\title{
Kratzer Potential for Vibrational Levels in Molecular Hydrogen
}

\author{
Guido Van Hooydonk \\ Ghent University, Faculty of Sciences, Krijgslaan 281, B-9000 Ghent, Belgium \\ Reprint requests to G. V. H.; E-mail: guido.vanhooydonk@ugent.be
}

Z. Naturforsch. 64a, 801 - 810 (2009); received November 22, 2008 / revised March 10, 2009

\begin{abstract}
The vibrational part of the dihydrogen Hamiltonian obeys a quantized Sommerfeld-Kratzer potential, which takes into account internal $\mathrm{H}_{2}$ symmetries. All constants $\omega_{\mathrm{e}}, k_{\mathrm{e}}$, and $r_{\mathrm{e}}$ needed for the $\mathrm{H}_{2}$ vibrational system derive from hydrogen mass. Ionic Kratzer bond theory gives covalent bond energy within $0.08 \%$ and all levels within $0.02 \%$, which is 30 times better than with a Dunham oscillator and as accurate as early ab initio quantum mechanics.
\end{abstract}

Key words: Hydrogen Molecule; Kratzer Potential; Vibrational Levels.

PACS numbers: 11.30.-j; 31.10.+z; 34.20.-b

\section{Introduction}

Physicists focus on the simple line spectrum of atom $\mathrm{H}$ with fine and hyperfine structure and less on the more complex band spectrum of molecule $\mathrm{H}_{2}$ [1]. Since Bohr's simple, fairly accurate atom theory made $\mathrm{H}$ prototypical for atomic spectroscopy, a simple bond theory should make $\mathrm{H}_{2}$ prototypical for molecular spectroscopy [2]. However, only a complex quantum mechanics (QM) theory can account accurately for $\mathrm{H}_{2}$ levels and its potential energy curve (PEC) $[3,4]$. During the last decades, the rigour of ab initio QM has been abandoned in favour of density functional theory (DFT) while simpler Bohr-type bond theories remain of interest [5]. Since for both $\mathrm{H}$ and $\mathrm{H}_{2}$, the role of internal symmetries is still uncertain, we expand on these symmetries for $\mathrm{H}_{2}$.

By its complexity, QM also fails on a simple analytical function for PECs, i. e. a low parameter universal function (UF) [2, 6, 7], needed to unify shape-invariant, asymmetric PECs [2]. This justifies many attempts to find a UF, usually dealt with using Dunham theory [8]. If $\mathrm{H}_{2}$ is supposed to be the best starting point to get at universal behaviour, its vibrational levels must be understood with a simple low-order oscillator like Dunham's or Kratzer's, a thesis we develop here $[2,6]$.

Since anharmonicity flaws the harmonic oscillator (HO), which is important for physics [9], we confront it with the $\mathrm{H}_{2}$ spectrum in Section 2. Dunham and Kratzer oscillators are in Section 3. In Section 4, the three $\mathrm{H}_{2}$ parameters $r_{0}, \omega_{\mathrm{e}}$, and $k_{\mathrm{e}}$ are derived solely from mass $m_{\mathrm{H}}$. In Section 5 , effects of $P$ and $C$ for $\mathrm{H}_{2}$ are quantified and old quantum theory leads to a quantized Kratzer bond theory. The accuracy of Dunham and Kratzer oscillators in Section 6 not only shows that Kratzer's is superior to Dunham's but also that a Kratzer theory is as accurate as earlier ab initio QM [10]. Internal $\mathrm{H}_{2}$ symmetries are discussed in Section 7. Implications for the theory of the chemical bond are in Section 8. Section 9 concludes.

\section{Quantum Harmonic Oscillator and Anharmonicity in Bond $\mathrm{H}_{2}$}

$\mathrm{H}_{2}$ rotator-vibrator levels $E_{\mathrm{V}, \mathrm{J}}$ vary with vibrational and rotational quantum numbers $v$ and $J$. For vibrational levels $v(J=0)$, Schrödinger's quantum harmonic oscillator (HO) [11] gives equally spaced levels with

$$
E_{\mathrm{v}+1 / 2}=\omega_{\mathrm{e}}(v+1 / 2) \text { or } E_{\mathrm{v}+1 / 2} / \omega_{\mathrm{e}}=v+1 / 2,
$$

where $\omega_{\mathrm{e}}$ is the fundamental vibrational frequency. Nevertheless, (1) disagrees with the observed $\mathrm{H}_{2}$ anharmonicity. A series expansion in half integer $v$

$$
\begin{aligned}
E_{\mathrm{v}+1 / 2}= & \omega_{\mathrm{e}}(\mathrm{v}+1 / 2)-\omega_{\mathrm{e}} x_{\mathrm{e}}(v+1 / 2)^{2} \\
& +\omega_{\mathrm{e}} y_{\mathrm{e}}(v+1 / 2)^{3}-\ldots
\end{aligned}
$$

gives a better agreement but this is equivalent with an expansion in integer $v$

$$
E_{\mathrm{v}}=A+B v+C v^{2}+D v^{3}-\ldots
$$


Table 1. Observed vibrational data for the $\mathrm{X}^{1} \Sigma_{\mathrm{g}}{ }^{1}$ state of $\mathrm{H}_{2}$ [12] (in $\mathrm{cm}^{-1}$ ).

\begin{tabular}{rcc}
\hline & $\begin{array}{c}\text { Vibrational differences } \\
(\text { quanta) }\end{array}$ & $\begin{array}{r}\text { Vibrational energies } \\
\text { or levels } E(v, 0) \\
\left(G_{0}(v) \text { in }[12]\right)\end{array}$ \\
\hline 0 & $(\Delta G(v+1 / 2)$ in $[12])$ & 0.00 \\
1 & 4402.93 & 4161.14 \\
2 & 4161.14 & 8086.93 \\
3 & 3925.79 & 11782.36 \\
4 & 3695.43 & 15250.31 \\
5 & 3467.95 & 18491.92 \\
6 & 3241.61 & 21505.78 \\
7 & 3013.86 & 24287.91 \\
8 & 2782.13 & 26831.16 \\
9 & 2543.25 & 29124.09 \\
10 & 2292.93 & 31150.47 \\
11 & 2026.38 & 32887.13 \\
12 & 1736.66 & 34302.20 \\
13 & 1415.07 & 35351.36 \\
14 & 1049.16 & 35973.38 \\
\hline
\end{tabular}

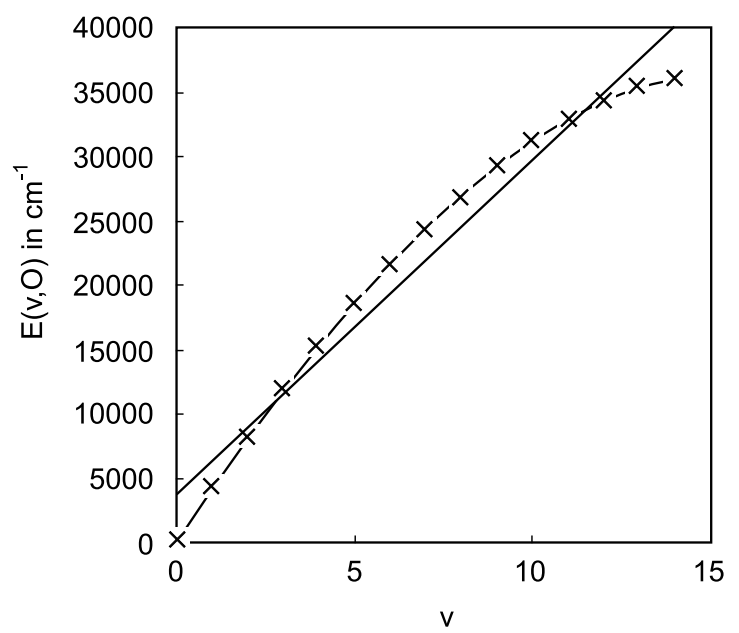

Fig. 1. Plot of 14 vibrational levels $E(v, 0)[12]$ versus $v$. Linear fit (straight line); second-, fourth-, and sixth-order fits coalesce to a single broad curve $(-x-)$.

Coefficients $A, B, C, \ldots$ derive from those in (2), e.g. $A=1 / 2 \omega_{\mathrm{e}}\left(1-x_{\mathrm{e}}+y_{\mathrm{e}}-\ldots\right) \ldots .$. Figure 1 gives the $E_{\mathrm{v}}(v)$ plot for all 14 observed $\mathrm{H}_{2}$-levels in Table 1 [12]. The first-, second-, fourth-, and sixth-order fits give errors of respectively $1839.93,111.84,7.15$, and $0.24 \mathrm{~cm}^{-1}$. In second-order fit, $E_{\mathrm{v}}=[-161.11+4397.26 v-$ $\left.128.19 v^{2}\right] \mathrm{cm}^{-1}$, suggested by Morse [13,14], gives errors of $112 \mathrm{~cm}^{-1}$. Even a sixth-order fit is not of spectroscopic accuracy. With errors of $3.2 \mathrm{~cm}^{-1}$, earlier ab initio QM [10] ranks between fourth- and sixth-order fits. With errors of $1840 \mathrm{~cm}^{-1}$, (1) obviously fails for the simplest and stable vibrator in nature, $\mathrm{H}_{2}$.
Using reduced mass, equilibrium separation $r_{0}$, and kinetic energy $T=1 / 2 \mu v_{0}^{2}=1 / 2 \mu \omega_{\mathrm{e}}^{2} r_{0}^{2}$, the vibrator energy $E_{0}=T_{0}+V_{0}$ depends on $V_{0}$, according to $-1 / 2 k_{\mathrm{e}} r_{0}^{2}$ (Hooke, Dunham) or $-1 / 2 e^{2} / r_{0}$ (Coulomb). For $E_{0}=0$, the fundamental frequency $\omega_{\mathrm{e}}$ is available with the equations

$$
\begin{aligned}
& \text { Hooke, Dunham: } \\
& \qquad \begin{array}{c}
E_{0}=1 / 2 \mu_{\mathrm{H}} \omega_{\mathrm{e}}{ }^{2} r_{0}{ }^{2}-1 / 2 k_{\mathrm{e}} r_{0}^{2}=0 \\
\mu_{\mathrm{H}} \omega_{\mathrm{e}}{ }^{2}=k_{\mathrm{e}} \text { or } \omega_{\mathrm{e}}=\sqrt{ }\left(k_{\mathrm{e}} / \mu_{\mathrm{H}}\right)
\end{array}
\end{aligned}
$$

Coulomb:

$$
\begin{aligned}
& E_{0}=1 / 2 \mu_{\mathrm{H}} \omega_{\mathrm{e}}{ }^{2} r_{0}{ }^{2}-1 / 2 e^{2} / r_{0}=0 \\
& k_{\mathrm{e}}=e^{2} / r_{0}{ }^{3} ; \\
& \mu_{\mathrm{H}} \omega_{\mathrm{e}}^{2}=e^{2} / r_{0}{ }^{3} \text { or } \omega_{\mathrm{e}}=\sqrt{ }\left[e^{2} /\left(\mu_{\mathrm{H}} r_{0}^{3}\right)\right] .
\end{aligned}
$$

Hooke's equation (4) gives a standard $\mathrm{HO}$ relation but fails to identify $k_{\mathrm{e}}$. Coulomb's equation (5) identifies $k_{\mathrm{e}}$ as

$$
k_{\mathrm{e}}=e^{2} / r_{0}^{3}
$$

giving $\omega_{\mathrm{e}}=4390 \mathrm{~cm}^{-1}$ as observed (see below). With Dunham's coefficient $a_{0}=1 / 2 k_{\mathrm{e}} r_{0}^{2} \quad[2,7,8]$, all asymptotes converge to ionic bond energy $D_{\text {ion }}$ :

$$
\begin{aligned}
V\left(r_{0}\right) & =a_{0}=1 / 2 k_{\mathrm{e}} r_{0}^{2}=1 / 2 e^{2} / r_{0}=1 / 2 D_{\text {ion }} \\
& \approx 79000 \mathrm{~cm}^{-1} .
\end{aligned}
$$

The only asymptote missing in (7) is the covalent bond energy $D_{\mathrm{e}}=36118.3 \mathrm{~cm}^{-1}$ (see Section 6.3), which is much smaller than (7), but the standard for scaling the spectroscopic constants [2,7]. With $(5)-(6)$, it is evident that, if $r_{0}$ for $\mathrm{H}_{2}$ is available classically, $\omega_{\mathrm{e}}$ or $k_{\mathrm{e}}$ are available too (Section 6).

\section{Revisiting the HO: Dunham and Kratzer Potentials}

Sinusoidal solutions for HO (1) derive from Hooke's force $F=-k_{\mathrm{e}} r$ and Newton's second law $F=m a$ [11]. With $V(r)=1 / 2 k_{\mathrm{e}} r^{2}$, a Hooke-Dunham HO potential behind (4)

$V_{\mathrm{HO}}=1 / 2 k_{\mathrm{e}}\left(r-r_{0}\right)^{2}=1 / 2 k_{\mathrm{e}} r_{0}^{2}\left(r / r_{0}-1\right)^{2}=a_{0} d_{\mathrm{D}}^{2}$

is so firmly entrenched that alternatives are rarely employed, although it is wrong $[2,15]$. Variable

$$
d_{\mathrm{D}}=\left(r / r_{0}-1\right) \text { or } r / r_{0}=1+d_{\mathrm{D}}
$$


transforms (8) in $V_{\mathrm{HO}}=a_{0} d_{\mathrm{D}}^{2}$, where $a_{0}=1 / 2 k_{\mathrm{e}} r_{0}{ }^{2}$. $V_{\mathrm{HO}} / a_{0}=d_{\mathrm{D}}^{2}$ has the solutions $\pm d_{\mathrm{D}}$ for the $r$ dependence in non-convergent, symmetric PECs. Even Dunham's more flexible series expansion

$$
V_{\mathrm{HO}}=a_{0} d_{\mathrm{D}}^{2}\left(1+a_{1} d_{\mathrm{D}}+a_{2} d_{\mathrm{D}}^{2}+\ldots\right)
$$

faces convergence problems [7]. Asymmetric PECs, convergent without expansions, use alternatives:

$$
\begin{aligned}
d_{\mathrm{SK}} & =\left(1-r_{0} / r\right)=\left[1-1 /\left(1+d_{\mathrm{D}}\right)\right] \\
& =d_{\mathrm{D}} /\left(1+d_{\mathrm{D}}\right), \\
V_{\mathrm{SK}} & =1 / 2 k_{\mathrm{e}} r_{0}^{2}\left(1-r_{0} / r\right)^{2}=a_{0}\left(1-r_{0} / r\right)^{2} \\
& =1 / 2\left(e^{2} / r_{0}\right)\left(1-r_{0} / r\right)^{2},
\end{aligned}
$$

respectively, which is the dimensionless Sommerfeld ${ }^{1}$ Kratzer variable (11) and potential (12) $[2,15]$. Reduced $V_{\mathrm{SK}} / a_{0}$ gives the solutions $\pm d_{\mathrm{SK}}$ for asymmetric, convergent PECs $[2,7,15]$ but is ionic (see further below). For a classical approach to $\mathrm{H}_{2}$ based on (12), $r_{0}$ must be available with classical physics.

\section{Classical Equilibrium Separation $\boldsymbol{r}_{0}$ in $\mathbf{H}_{2}$}

An independent classical calculus of $r_{0}$ will lead to a classical $\omega_{\mathrm{e}}$ using

$$
\omega_{\mathrm{e}}=\sqrt{ }\left(k_{\mathrm{e}} / \mu\right) /(2 \pi c)=\sqrt{ }\left[e^{2} /\left(\mu r_{0}^{3}\right)\right] /(2 \pi c) .
$$

The only classical formula available for spherical point-like particles with mass $m_{x}$ is

$$
m_{x}=(4 \pi / 3) \gamma_{x} r_{x}^{3}
$$

with $\gamma_{x}$, the density $\left(\mathrm{g} / \mathrm{cm}^{3}\right)$ and $4 \pi / 3$, the spherical form factor, applicable for two spheres in a $\mathrm{H}_{2}$ dumbbell. Questions emerge for micro-systems: (a) Is (14) adequate for $\mathrm{H}_{2}$ concerning form factor and density; (b) Is $2 m_{\mathrm{H}}$ or reduced mass $1 / 2 m_{\mathrm{H}}$ needed?; (c) Do results apply for $r_{0}$ in $D_{\text {ion }}=e^{2} / r_{0}$ or for $2 r_{0}$ in $a_{0}=$ $1 / 2 k_{\mathrm{e}} r_{0}^{2}=1 / 2 e^{2} / r_{0}$, all appearing in (7)?

The sum of electron and proton mass [21] is $m_{\mathrm{H}}=$ $1 /\left(5.97538 .10^{23}\right) \mathrm{g}$. With $\gamma_{\mathrm{H}}=1 \mathrm{~g} / \mathrm{cm}^{3},(14)$ gives

$$
\begin{aligned}
r_{\mathrm{H}}=\left[\left(3 m_{\mathrm{H}}\right) /\left(4 \pi \gamma_{\mathrm{H}}\right)\right]^{1 / 3} & =7.36516 \cdot 10^{-9} \mathrm{~cm} \\
& =0.736516 \AA
\end{aligned}
$$

\footnotetext{
${ }^{1}$ Sommerfeld first used (11) for $\mathrm{H}[16,17]$. His pupil Kratzer used it for a general bond theory [18] and their colleague Kossel [19] for an ionic bond theory. Fues [20] solved the wave equation for (12).
}

as classical radius $r_{\mathrm{H}}$, whereas $r_{\mathrm{B}}=0.529177 \AA$. Since $r_{\mathrm{HH}}=2 r_{\mathrm{H}}, r_{0}$ is $r_{\mathrm{HH}}=1.473032 \AA$, which is typical for a virial rather than a Coulomb energy (observed $r_{0}=$ $r_{\mathrm{HH}}=0.740144 \AA$ [22]), if $\gamma_{x}=1 \mathrm{~g} / \mathrm{cm}^{3}$ makes sense ${ }^{2}$.

The classical result $r_{0}$ (15) immediately gives (i) with (13), the fundamental $\mathrm{H}_{2}$ vibrational frequency $^{3}$, equal to

$$
\omega_{\mathrm{e}}=4410.1722 \mathrm{~cm}^{-1}
$$

where $4402.93 \mathrm{~cm}^{-1}$ [12] or $4401.213 \mathrm{~cm}^{-1}$ [22] are observed; (ii) with (7), a virial energy for $\mathrm{H}_{2}$, equal to half the ionic bond energy $D_{\text {ion }}$ or

$$
-V_{0}=e^{2} /\left(2 r_{\mathrm{H}}\right)=a_{0}=78844.9125 \mathrm{~cm}^{-1}
$$

(observed $a_{0}=1 / 2 k_{\mathrm{e}} r_{0}^{2} \approx 79000 \mathrm{~cm}^{-1} \quad$ [24]); (iii) with the same dimension for (16)-(17), a quantum hypothesis emerges. The small ratio of step $\omega_{\mathrm{e}} \sim 4400 \mathrm{~cm}^{-1}$ (16) and total gap $a_{0} \sim 79000 \mathrm{~cm}^{-1}$ (17) shows that a number steps $v$ as in $(1)-(3)$ is needed to cover this gap. This ratio is the number

$$
\begin{aligned}
q=\omega_{\mathrm{e}} / a_{0} & =4410.1722 / 78844.9125 \\
& =0.05593477
\end{aligned}
$$

which can bring in quantization following step $\delta_{\mathrm{v}}$, function of $v$. Hence, bond quantum hypothesis

$$
r / r_{0}-1=\Delta / r_{0}=d_{\mathrm{HO}}=\delta_{\mathrm{v}}=q v
$$

must be plugged in variable $d_{\mathrm{HO}}$ and $d_{\mathrm{SK}}$ for potentials $V_{\mathrm{HO}}$ and $V_{\mathrm{SK}}$ (see Section 6). With (15), mass $m_{\mathrm{H}}$ indeed provides with three fundamental parameters $\omega_{\mathrm{e}}$, $r_{0}$, and $k_{\mathrm{e}}$ for bond $\mathrm{H}_{2}$.

\section{Kratzer Bond Theory. Connection with $C, P$, and $\mathrm{CP}$ in $\mathrm{H}_{2}$}

\subsection{Vibrational $\mathrm{H}_{2}$ Energy and CP Symmetries}

The Hamiltonian $\mathbf{H}$ (or energy $E$ ) for four-particle system $\mathrm{H}_{2}$ (with pairs of charge-conjugated leptons $\mathrm{a}, \mathrm{b}$

\footnotetext{
${ }^{2}$ For systems with constant $m_{x} / \gamma_{x}$, all $r_{x}$ are (nearly) equal, as observed for isotopomers $\mathrm{H}_{2}, D_{2}$, and $T_{2}$ [23].

${ }^{3}$ The same formula for an electron $\left(m_{\mathrm{e}}=m_{\mathrm{H}} / 1837.15267\right.$ and radius $r_{\mathrm{B}}$ ) gives $\omega_{\mathrm{e}}=219474.65 \mathrm{~cm}^{-1}=2 \cdot 109737.31 \mathrm{~cm}^{-1}$, or twice the Rydberg $e^{2} / r_{\mathrm{B}}$ [21]. This shows how the internal mechanics of $\mathrm{H}$ and $\mathrm{H}_{2}$ are intimately connected.
} 
and nucleons A, B) consists of four kinetic and six as potential energy terms [24]:

$$
\begin{aligned}
\mathbf{H}= & 1 / 2 p_{\mathrm{a}}^{2} / m_{\mathrm{a}}+1 / 2 p_{\mathrm{b}}^{2} / m_{\mathrm{b}}+1 / 2 p_{\mathrm{A}}{ }^{2} / m_{\mathrm{A}} \\
& +1 / 2 p_{\mathrm{B}}{ }^{2} / m_{\mathrm{B}}-e^{2} / r_{\mathrm{aA}}-e^{2} / r_{\mathrm{bB}}-e^{2} / r_{\mathrm{bA}} \\
& -e^{2} / r_{\mathrm{aB}}+e^{2} / r_{\mathrm{ab}}+e^{2} / r_{\mathrm{AB}} .
\end{aligned}
$$

With $n, v$, and $J$ for electronic $E_{\text {elec }}$, vibrational $E_{\text {vib }}$, and rotational energies $E_{\text {rot }}$, total energy obeys $E=$ $E_{\text {elec }}+E_{\mathrm{vib}}+E_{\mathrm{rot}}=E_{\mathrm{n}}+E_{\mathrm{v}}+E_{\mathrm{J}}$. Subtracting ${ }^{4} E_{0}=$ $E_{\mathrm{n}}=1 / 2 m_{\mathrm{a}} v_{\mathrm{a}}^{2}+1 / 2 m_{\mathrm{b}} v_{\mathrm{b}}^{2}-e^{2} / r_{\mathrm{aA}}-e^{2} / r_{\mathrm{bB}}$ and neglecting $E_{\mathrm{rot}}=E_{\mathrm{J}}$, (20) becomes $E_{\mathrm{vib}}=E-E_{0} \approx$ $1 / 2 m_{\mathrm{A}} v_{\mathrm{A}}^{2}+1 / 2 m_{\mathrm{B}} v_{\mathrm{B}}^{2}-e^{2} / r_{\mathrm{bA}}-e^{2} / r_{\mathrm{aB}}+e^{2} / r_{\mathrm{ab}}+$ $e^{2} / r_{\mathrm{AB}}$. Notations $r=r_{\mathrm{bA}}=r_{\mathrm{Ba}}$ and $m v^{2}=m_{\mathrm{A}} v_{\mathrm{A}}^{2}=$ $m_{\mathrm{B}} v_{\mathrm{B}}^{2}\left(m=1836.15 m_{\mathrm{e}}\right.$ close to $\left.m_{\mathrm{H}}=1837.15 m_{\mathrm{e}}\right)$ lead to $\Delta E=\Delta T+\Delta V$ or

$$
\begin{aligned}
\Delta E & =E-E_{0} \\
& \approx 2\left(1 / 2 m v^{2}\right)-2 e^{2} / r+\left(e^{2} / r_{\mathrm{AB}}\right)\left(1+r_{\mathrm{AB}} / r_{\mathrm{ab}}\right) \\
& =m v^{2}+\left(e^{2} / r_{\mathrm{AB}}\right)\left[\left(1+r_{\mathrm{AB}} / r_{\mathrm{ab}}-2 r_{\mathrm{AB}} / r\right)\right] \\
& \equiv m v^{2}+\left(e^{2} / r_{\mathrm{ab}}\right)\left[\left(1+r_{\mathrm{ab}} / r_{\mathrm{AB}}-2 r_{\mathrm{ab}} / r\right)\right] \\
& \equiv m v^{2}+\left(e^{2} / r\right)\left[\left(r / r_{\mathrm{AB}}+r / r_{\mathrm{ab}}-2\right)\right],
\end{aligned}
$$

where all three composite Coulomb terms $\Delta V$ are equal. For large $r_{\mathrm{AB}}, r_{\mathrm{AB}} \approx r_{\mathrm{ab}}$ gives $\Delta E=E-E_{0} \approx$ $m v^{2}+2 e^{2}\left(1 / r_{\mathrm{AB}}-1 / r\right)$. With $r_{0}$ and $r_{\mathrm{AB}}=r_{1}=a_{1} r_{0}$, $r=r_{2}=a_{2} r_{0}$ and factor $1 / 2$, we get

$$
\begin{aligned}
1 / 2 \Delta E & \approx 1 / 2 m v^{2}+\left(e^{2} / r_{0}\right)\left(r_{0} / r_{\mathrm{AB}}-r_{0} / r\right) \\
& =1 / 2 m v^{2}+\left(e^{2} / r_{0}\right)\left(r_{0} / r_{1}-r_{0} / r_{2}\right) \\
& =1 / 2 m v^{2}+\left(e^{2} / r_{0}\right)\left(1 / a_{1}-1 / a_{2}\right),
\end{aligned}
$$

a simple result for $E_{\mathrm{vib}}$ (see also Section 6.1). To proceed with all equivalent variants in (21), the most appropriate scaling aid must be found. Since nucleons have the greater inertia, we prefer scaling by $r_{\mathrm{AB}}$, which appears in ionic bonding, is important in BO (Born-Oppenheimer) approximations and is the common variable for PECs [24].

Magnitude and sign of Coulomb terms in (21) depend on the configuration (geometry) of $\mathrm{H}_{2}$, which is subject to internal symmetries [25]. The ionic solution $-e^{2} / r_{\mathrm{AB}}$ for (21) would require either that coefficient $\left(1+r_{\mathrm{AB}} / r_{\mathrm{ab}}-2 r_{\mathrm{AB}} / r\right)$ for $e^{2} / r_{\mathrm{AB}}$ in (21) is not only constant but also negative. Its final sign depends on geometry related operator parity $\mathbf{P}^{2}=1$ or geometry independent charge operator $\mathbf{C}^{2}=1$ [25]. To accommodate for this algebra, we use a form factor $\pm A_{\mathrm{r}}$, defined

\footnotetext{
${ }^{4}$ The use of $v$ for vibrational quantum number as well as for velocity must be clear from the context given.
}

$$
\pm A_{\mathrm{r}}= \pm\left(1+r_{\mathrm{AB}} / r_{\mathrm{ab}}-2 r_{\mathrm{AB}} / r\right) .
$$

Its subscript $r$ refers to scaling by $r_{\mathrm{AB}}$, its magnitude to the $\mathrm{H}_{2}$ geometry, and its sign \pm to the discrete values allowed by internal symmetry $C$. Energy (21) rewritten as

$$
\begin{aligned}
E_{\mathrm{vib}} & =\Delta E\left(=\Delta \mathbf{H}^{\prime}=p^{2} / m \pm A_{\mathrm{r}} e^{2} / r_{\mathrm{AB}}\right) \\
& =+m v^{2} \pm A_{\mathrm{r}} e^{2} / r_{\mathrm{AB}}
\end{aligned}
$$

has positive kinetic energy $T$ and positive or negative potential energy $V$, but this algebra must be in line with classical physics. Only attractive $-A_{\mathrm{r}} e^{2} / r_{\mathrm{AB}}$ can give a stable bond, repulsive $+A_{\mathrm{r}} e^{2} / r_{\mathrm{AB}}$ cannot $^{5}[25,26]$.

Proceeding with (24) and its periodic motion (for vibration and rotation, see Section 4.2), angular velocity $\omega$, velocity $v=\omega r$, and angular momentum $p=m_{\mathrm{H}} v=m_{\mathrm{H}} \omega r$ lead to the classical equation:

$$
\Delta E=+m_{\mathrm{H}} \omega^{2} r_{\mathrm{AB}}^{2} \pm A_{\mathrm{r}} e^{2} / r_{\mathrm{AB}} \text {. }
$$

This cannot only be interpreted by vibrations $\omega^{2}=$ $A_{\mathrm{r}} e^{2} /\left(\mu_{\mathrm{H}} r^{3}\right)$ like (7) but also by rotations obeying Kepler's third law $\omega^{2} r_{0}{ }^{3}=A_{\mathrm{r}} e^{2} / \mu_{\mathrm{H}}=C$. For rotations coupled to vibrations, old quantum theory gives

$p_{1}=f \hbar / r_{1}, \quad p_{2}=f \hbar / r_{2}$, and $p_{\mathrm{HH}}=f \hbar / r_{\mathrm{HH}}$,

where $f$ is a field factor. With Bohr's theory as guideline, all relations and identities easily follow:

$$
\begin{array}{ll}
\text { (a) } & 2 m_{\mathrm{H}} \omega^{2} r^{3}=2 m_{\mathrm{H}} v^{2} r=A_{\mathrm{r}} e^{2} \text { or } \\
& v^{2}=1 / 2 A_{\mathrm{r}} e^{2} /\left(m_{\mathrm{H}} r\right), \\
\text { (b) } & 2 m_{\mathrm{H}} \omega r^{2}=2 m_{\mathrm{H}} v r=2 p_{\mathrm{H}} r=f \hbar \text { or } \\
& v=1 / 2 f \hbar /\left(m_{\mathrm{H}} r\right), \\
\text { (c) } \quad & v=A_{\mathrm{r}} e^{2} /(f \hbar) \text { and } \\
& r=1 / 2\left(f^{2} / A_{\mathrm{r}}\right)\left(\hbar^{2} /\left(m_{\mathrm{H}} e^{2}\right) .\right.
\end{array}
$$

For $A_{\mathrm{r}}=1$, the field scale factor $f$ in (27) can be identified by using the classical $r_{0}(15)$ :

$$
\begin{aligned}
& r_{0}=1 / 2 f^{2} \hbar^{2} /\left(m_{\mathrm{H}} e^{2}\right) \sim\left[\left(3 m_{\mathrm{H}} /\left(4 \pi \gamma_{\mathrm{H}}\right)\right]^{1 / 3}\right. \text { and } \\
& f \sim m_{\mathrm{H}}^{2 / 3}(\hbar / e)[3 /(4 \pi \gamma)]^{1 / 6} \sim 1 / q
\end{aligned}
$$

in agreement with (18), pending corrections for reduced mass and the virial.

\footnotetext{
${ }^{5}$ Ionic $-e^{2} / r_{\mathrm{AB}}$ is only suspicious for two neutral atoms when $r_{\mathrm{AB}} \gg r_{\text {crit }}$, with $r_{\text {crit }}$ the critical distance, where ionic and non-ionic PECs cross [25]. At closer range, particle transfers can occur. Generically, ionic $-A_{\mathrm{r}} e^{2} / r_{\mathrm{AB}}$ is also obtained, independent of geometry or without particle transfer, by charge inversion symmetry $C$ in one atom [25].
} 
With (26), kinetic energy $T=p_{\mathrm{H}}^{2} / m_{\mathrm{H}}=\left(f^{2} \hbar^{2} /\right.$ $\left.m_{\mathrm{H}}\right) / r^{2}$ can now be plugged in (24). The result is

$$
\begin{aligned}
\Delta \mathbf{H}^{\prime} & = \pm A_{\mathrm{r}} e^{2} / r+m_{\mathrm{H}} \omega^{2} r^{2} \\
& = \pm A_{\mathrm{r}} e^{2} / r+\left(f^{2} \hbar^{2} / m_{\mathrm{H}}\right) / r^{2} \\
& = \pm A_{\mathrm{r}} e^{2} / r+\left[f^{2} \hbar^{2} /\left(m_{\mathrm{H}} e^{2}\right)\right]\left(e^{2} / r^{2}\right) .
\end{aligned}
$$

Using constants $A=A_{\mathrm{r}} e^{2}$ and $B=e^{2} f^{2} \hbar^{2} /\left(m_{\mathrm{H}} e^{2}\right)$, this is of general Kratzer form:

$$
V(r)=A / r+B / r^{2} .
$$

The equilibrium $\mathrm{d} V / \mathrm{d} r$ at $r_{0}$, gives $B=-1 / 2 A r_{0}$ for the stable solution $\mathrm{V}_{\mathrm{SK}}\left(r_{0}\right)<0$, since $B / r^{2}>0$. We get

$$
\begin{aligned}
& \Delta \mathbf{H}^{\prime}=-A_{\mathrm{r}} e^{2} / r+1 / 2 A_{\mathrm{r}} e^{2} / r^{2} \text { or } \\
& \begin{aligned}
\Delta^{\prime \prime} \mathbf{H} & =\Delta \mathbf{H}^{\prime}+1 / 2 A_{\mathrm{r}} e^{2} / r_{0} \\
& =+\left(1 / 2 A_{\mathrm{r}} e^{2} / r_{0}\right)\left(1-r_{0} / r\right)^{2}=V_{\mathrm{SK}},
\end{aligned}
\end{aligned}
$$

the Sommerfeld-Kratzer Coulomb potential (12) for $-A_{\mathrm{r}}=-1$. Quantum theory gives solution (31) or (12) for vibrational $\mathrm{H}_{2}$ levels obeying a Kratzer, not a Dunham oscillator [2,25].

The second derivative $d^{2} / d r^{2}$ of (29) for the force constant equations leads to

$$
\omega_{\mathrm{e}}^{2}=A_{\mathrm{r}} e^{2} /\left(m_{\mathrm{H}} r_{0}^{3}\right) ; \quad k_{\mathrm{e}}=A_{\mathrm{r}} e^{2} / r_{0}^{3},
$$

which is impossible with Hooke's law for Schrödinger's HO (1), see Section 2. The reduced mass $\mu$ for $\mathrm{H}_{2}$,

$$
\begin{aligned}
\mu & =m_{\mathrm{H}} m_{\mathrm{H}} /\left(m_{\mathrm{H}}+m_{\mathrm{H}}\right) \\
& =m_{\mathrm{H}} /\left(1+m_{\mathrm{H}} / m_{\mathrm{H}}\right)=1 / 2 m_{\mathrm{H}},
\end{aligned}
$$

gives the scale factor $s=1 / 2$ for dimer $H_{2}=H_{\mathrm{a}} H_{\mathrm{B}}$. The dimensionless recoil correction in $A B\left(m_{\mathrm{A}}, m_{\mathrm{B}}\right)$ is

$$
s=1 /\left(1+m_{\mathrm{a}} / m_{\mathrm{B}}\right) .
$$

Following Section 2, $A_{\mathrm{r}} e^{2}$ simplified to $e^{2}$ means that the inter-atomic field is ionic [27]. At $r_{0}$

$$
k_{\mathrm{e}}=A_{\mathrm{r}} e^{2} / r_{0}^{3} \equiv e^{2} / r_{0}^{3}
$$

implies that the Coulomb attraction $-e^{2} / r_{0}$, i. e. ionic bond energy $D_{\text {ion }}$, appears for covalent $\mathrm{H}_{2}$ [2,27]. We found [27] that plugging observed $r_{0}=0.74 \AA$ [22] in (35) returns observed $k_{\mathrm{e}}=5.7 .10^{5}$ dyne $/ \mathrm{cm}$ and $\omega_{\mathrm{e}} \approx 4400 \mathrm{~cm}^{-1}$ for $\mathrm{H}_{2}$ [12,22], see Section 2 .

\subsection{Quantum Hypothesis for $\mathrm{H}_{2}$ Vibrations and} Connection with Intra-Molecular Rotations

Like (25)-(27), the angular velocity $v_{\mathrm{e}}$ for a rotating electron $m_{\mathrm{e}}$ in $\mathrm{H}$ derives from the ratio of radial equilibrium condition $m_{\mathrm{e}} v_{\mathrm{e}}^{2} / r=e^{2} / r^{2}$ and quantum rule for angular momentum $m v_{\mathrm{e}} r=p r=n \hbar$, or

$$
v_{\mathrm{e}}=m_{\mathrm{e}} v_{\mathrm{e}}^{2} r / m_{\mathrm{e}} v_{\mathrm{e}} r=e^{2} /(n \hbar)=\alpha c / n
$$

without recoil. Using Bohr radius $r_{\mathrm{B}}$ and $\mathrm{H}$-size differences $\Delta_{\mathrm{H}}$

$$
\begin{aligned}
& r=e^{2} / m_{\mathrm{e}} v_{\mathrm{e}}^{2}=n^{2} \hbar^{2} /\left(m_{\mathrm{e}} e^{2}\right)=n^{2} r_{\mathrm{B}}, \\
& \Delta_{\mathrm{H}}=r-r_{\mathrm{B}}=r_{\mathrm{B}}\left(n^{2}-1\right)
\end{aligned}
$$

are incompatible with the linear rule (26). Quantization of the field by $1 / n$ instead of the angular momentum by $n$, gives the same rotational energies for $\mathrm{H}, E_{\mathrm{n}}=$ $-R_{\mathrm{H}} / n^{2}$, since

$$
v_{\mathrm{e}}=m_{\mathrm{e}} v_{\mathrm{e}}^{2} r / m_{\mathrm{e}} v_{\mathrm{e}} r=\left(e^{2} / n\right) / \hbar
$$

is identical with (36). Unlike (37), field quantization $1 / n$ brings in a linear $n$-dependence

$$
r=\hbar /\left(m_{\mathrm{e}} v_{\mathrm{e}}\right)=n \hbar^{2} /\left(m_{\mathrm{e}} e^{2}\right)=n r_{\mathrm{B}}
$$

instead of a quadratic $n^{2}$-dependence in (37). Linearly quantized $r$-differences

$$
\Delta_{\mathrm{r}}=r-r_{\mathrm{B}}=(n-1) r_{\mathrm{B}}=\ell r_{\mathrm{B}}
$$

bring in Sommerfeld's secondary quantum number $\ell=$ $n-1$. With (40), $\mathrm{H}_{2}$ quantization needs $\Delta_{\mathrm{r}}=r-r_{0}=$ $(n-1) r_{0}$, a linear, instead of a quadratic integer quantum number. Reduced equivalent

$$
\Delta_{\mathrm{r}} / r_{0}=r / r_{0}-1=(n-1)=\ell
$$

validates quantum hypothesis for vibrations in bonds (19), if $\ell$ is denoted by the number $v$ in (1). With this information, we can apply rule (19) for any separation $r$.

\section{Quantization of Symmetric Linear and Asymmetric Inverse Field Shifts}

Quantum hypothesis (19) for inverse ${ }^{6}$ and linear Kratzer and Dunham variables gives:

\section{Kratzer:}

$r_{0} / r=r_{0} /\left(r_{0} \pm \Delta\right)=1 /\left(1 \pm \Delta / r_{0}\right)=1 /\left(1 \pm \delta_{\mathrm{r}}\right)$,

\footnotetext{
${ }^{6} \mathrm{H}_{\mathrm{a}}$ and $\mathrm{H}_{\mathrm{b}}$ in $\mathrm{H}_{2}=\mathrm{H}_{\mathrm{a}} \mathrm{H}_{\mathrm{b}}$ can be distinguished by mass $m_{\mathrm{a}}$ and $m_{\mathrm{b}}$ and sizes $r_{\mathrm{a}}$ and $r_{\mathrm{b}}$. As in a balance, this gives $m_{\mathrm{a}} r_{\mathrm{a}}=m_{\mathrm{b}} r_{\mathrm{b}}(=C)$,
} 
Dunham:

$r / r_{0}=\left(r_{0} \pm \Delta\right) / r_{0}=\left(1 \pm \Delta / r_{0}\right)=\left(1 \pm \delta_{\mathrm{r}}\right)$,

where $\delta_{\mathrm{r}}$ or $\delta_{\mathrm{v}}$ is the numerical equivalent of a step (25)-(27). Difference $\Delta$

$$
r-r_{0}=+\Delta=+1 / 2 \Delta-(-1 / 2 \Delta)
$$

is distributed in an anti-symmetric way, i.e. left and right to the center of mass, placed at the origin, but equal in absolute magnitude and based on the arithmetic average. In terms of symmetries, (43) gives symmetric $\mathrm{H}_{2}$. However, symmetric (43) is different in Dunham and Kratzer variables (42).

\subsection{The v-Dependence: Different Analytical Form for Quantized Dunham and Kratzer Oscillators}

(i) The symmetric distribution (43), applied to Dunham's procedure for $r=r_{0} \pm \Delta$, gives

$$
r / r_{0}=1 \pm \Delta / r_{0}=\left(1 \pm \delta_{\mathrm{r}}\right)=\left(1 \pm \delta_{\mathrm{V}}\right),
$$

where left and right are avoided by virtue of (43). Dunham potential (8) away from $r_{0}$ becomes $1 / 2 k_{\mathrm{e}} r_{0}^{2}\left(r / r_{0}\right)^{2}=a_{0}\left(r / r_{0}\right)^{2}=a_{0}\left(1 \pm \delta_{\mathrm{r}}\right)^{2}$. With (19), reduced Dunham potential differences are

$$
\begin{aligned}
V_{\mathrm{Du}}^{\prime}-V_{0}^{\prime} & =\Delta V_{\mathrm{Du}}^{\prime}=-1 / 2(1-q v)^{2}+1 / 2 \\
& =+q v-1 / 2 q^{2} v^{2} .
\end{aligned}
$$

Using $\mathrm{a}_{0}(24)$ and $q(25)$, the numerical result of Dunham $\mathrm{H}_{2}$ theory in $\mathrm{cm}^{-1}$ is:

$$
\Delta V_{\mathrm{Du}}=\Delta E_{\mathrm{v}}=4410.17 v-123.34 v^{2} \mathrm{~cm}^{-1} .
$$

This is close to the observed second-order fit in Section 2 but with a large error of $111 \mathrm{~cm}^{-1}$. The improvement over HO (1) is considerable but a parameter for qv cannot reduce these errors.

where $\mathrm{C}$ is a constant, with dimensions $(\mathrm{e} / \mathrm{v})^{2}$, leading to recoil (34) using $m_{\mathrm{a}} / m_{\mathrm{b}}=r_{\mathrm{b}} / r_{\mathrm{a}}$. Hence, relations between $m_{x}$ and $r_{x}$ obey $m_{x}=C / r_{x}$ or $r_{x} / C=1 / m_{x}$. If $r_{\mathrm{HH}}$ required addition, reduced mass $\mu$ appears naturally

$r_{\mathrm{HH}}=\left(r_{\mathrm{a}}+r_{\mathrm{b}}\right)=C\left(1 / m_{\mathrm{a}}+1 / m_{\mathrm{b}}\right)=C\left(m_{\mathrm{a}}+m_{\mathrm{b}}\right) /\left(m_{\mathrm{a}} m_{\mathrm{b}}\right)=C / \mu$.

If mass $m_{\mathrm{HH}}$ required addition, reduced separation $\rho=r_{\mathrm{a}} r_{\mathrm{b}} /\left(r_{\mathrm{a}}+r_{\mathrm{b}}\right)$ appears naturally, too:

$$
m_{\mathrm{HH}}=m_{\mathrm{a}}+m_{\mathrm{b}}=C\left(1 / r_{\mathrm{a}}+1 / r_{\mathrm{b}}\right)=C\left(r_{\mathrm{a}}+r_{\mathrm{b}}\right) /\left(r_{\mathrm{a}} r_{\mathrm{b}}\right)=C / \rho .
$$

This explains (47) below since, if reduced separation is $\rho_{+}$by sum, reduced separation $\rho_{-}$by difference obeys

$$
1 / \rho_{-}=1 / r_{\mathrm{a}}-1 / r_{\mathrm{b}}=\left(r_{\mathrm{b}}-r_{\mathrm{a}}\right) / r_{\mathrm{a}} r_{\mathrm{b}} .
$$

(ii) Quantization for a Kratzer potential gives a problem with the anti-symmetric or left-right symmetric distribution (43). Inverse $r_{0} / r=r_{0} /(1 \pm \Delta)$ does not account for positions of $H_{\mathrm{a}}$ and $H_{\mathrm{b}}$ with respect to their common center, i. e. $\pm^{1 / 2} \Delta$ in (43). Equivalent rearrangements of the four inter-atomic Coulomb terms in (20) led to (22), where the composite the Coulomb $\operatorname{sum}\left(e^{2} / r_{0}\right)\left(r_{0} / r_{\mathrm{a}}-r_{0} / r_{\mathrm{B}}\right)$ gives similar inverse separations $1 / r_{\mathrm{A}}=1 /\left(r_{0}-1 / 2 \Delta\right)$ and $1 / r_{\mathrm{B}}=1 /\left(r_{0}+1 / 2 \Delta\right)$.

Applying these formally to a Kratzer variable gives

$$
\begin{aligned}
r_{0} / r_{\mathrm{A}}-r_{0} / r_{\mathrm{B}} & =r_{0}\left(r_{\mathrm{B}}-r_{\mathrm{A}}\right) / r_{\mathrm{A}} r_{\mathrm{B}} \\
& =1 /\left(1-1 / 2 \delta_{\mathrm{r}}\right)-1 /\left(1+1 / 2 \delta_{\mathrm{r}}\right) \\
& =\delta_{\mathrm{r}} /\left(1-1 / 4 \delta_{\mathrm{r}}^{2}\right) .
\end{aligned}
$$

Using (19), a more complex quantized $v$-dependence appears for a Kratzer theory,

$$
1 /(1-1 / 2 q v)-1 /(1+1 / 2 q v)=q v /\left(1-1 / 4 q^{2} v^{2}\right),
$$

instead of the simpler linear $q v$-dependence in Dunham's (45). The reduced Kratzer oscillator difference is

$$
\begin{aligned}
& \Delta V_{\mathrm{Kr}}^{\prime}=-1 / 2\left[1-q v /\left(1-1 / 4 q^{2} v^{2}\right)\right]^{2}+1 / 2= \\
& +q v /\left(1-1 / 4 q^{2} v^{2}\right)-1 / 2 q^{2} v^{2} /\left(1-1 / 4 q^{2} v^{2}\right)^{2}
\end{aligned}
$$

to be compared with Dunham's (45). In $\mathrm{cm}^{-1}$, the numerical Kratzer result is

$$
\begin{aligned}
\Delta V_{\mathrm{Kr}}= & \left(+4410.17 v-123.34 v^{2}-3.49 v^{3}\right) \\
& /\left(1-0.00078 v^{2}\right)^{2} \mathrm{~cm}^{-1} .
\end{aligned}
$$

Its accuracy is discussed in Section 6.2. Relation (50) gives higher-order terms in $v$ as in (2) - (3), to accommodate for anharmonicity. A parameter for $q v$ in (48) can affect goodness of fits.

Maximum $v_{0}$ for variable $q v$ gives $v / v_{0}$. For Dunham, $(1-x)^{2}=\left(1-v / v_{0}\right)^{2}$ and $x=v / v_{0}=0$ returns the well depth; $x=1$ or $v=v_{0}$ gives zero. For Kratzer $(1-y)^{2}, y=\left(v / v_{0}\right) /\left[1-1 / 4\left(v / v_{0}\right)^{2}\right]=$ $q v /\left[1-1 / 4(q v)^{2}\right], y=1$ implies that $q v=1-1 / 4(q v)^{2}$ or $v^{2}+4 v / q-4 / q^{2}=0$. Solving for $v_{0}$ gives

$$
\begin{aligned}
v_{0(\mathrm{Kr})} & =v_{0(\mathrm{Du})} /[1 / 2(1+\sqrt{ } 2)] \\
& =17.877967 / 1.207107=14.811,
\end{aligned}
$$

i. e. 14 levels as observed (see Table 1). The fact that band 15 is missing is in line with Kratzer theory (51) and is confirmed by the greater number of levels, observed for $D_{2}$ and $T_{2}[22,23]$. 
Table 2. Errors for $\mathrm{H}_{2}$ levels $E(v, 0)$ with Dunham (secondand fourth-order) and Kratzer (second-order) functions (in $\mathrm{cm}^{-1}$ ).

\begin{tabular}{rrrrr}
\hline $\mathrm{v}$ & Observed $E(v, 0)$ & \multicolumn{2}{c}{ Dunham } & Kratzer \\
& & second-order & fourth-order & second-order \\
\hline 0 & 0.00 & 161.11 & 8.08 & 3.57 \\
1 & 4161.14 & 53.18 & -8.34 & -3.91 \\
2 & 8086.93 & -33.74 & -8.43 & -3.94 \\
3 & 11782.36 & -94.63 & -1.67 & -0.77 \\
4 & 15250.31 & -126.64 & 5.20 & 2.45 \\
5 & 18491.92 & -128.61 & 8.56 & 4.15 \\
6 & 21505.78 & -101.96 & 7.09 & 3.59 \\
7 & 24287.91 & -50.66 & 1.75 & 1.13 \\
8 & 26831.16 & 18.13 & -4.81 & -2.02 \\
9 & 29124.09 & 92.98 & -9.39 & -4.48 \\
10 & 31150.47 & 157.65 & -8.71 & -4.51 \\
11 & 32887.13 & 188.98 & -1.59 & -1.37 \\
12 & 34302.20 & 155.08 & 9.30 & 4.04 \\
13 & 35351.36 & 11.66 & 13.69 & 6.80 \\
14 & 35973.38 & -302.54 & -10.72 & -4.73 \\
& absolute error & $\mathbf{1 1 1 . 8 4}$ & $\mathbf{7 . 1 5}$ & $\mathbf{3 . 4 3}$ \\
& \% error & $\mathbf{0 . 5 3 6}$ & $\mathbf{0 . 0 4 4}$ & $\mathbf{0 . 0 2 1}$ \\
\hline
\end{tabular}

\subsection{Normalization of Quantized Dunham and} Kratzer Oscillators. Formal Results

Dunham variable $\delta_{\text {Du }}$ and normalized Kratzer variable $\delta_{\mathrm{Kr}} / p$, respectively, equal to

$$
\begin{aligned}
& \delta_{\mathrm{Du}}=q v \\
& \delta_{\mathrm{Kr}} / p=(1 / p)[1 /(1-1 / 2 p q v)-1 /(1+1 / 2 p q v)] \\
&=q v /\left(1-1 / 4 p^{2} q^{2} v^{2}\right),
\end{aligned}
$$

imply normalizing factor 1 for qv in Dunham's (52) but $1 /\left(1-1 / 4 p^{2} q^{2} v^{2}\right)$ in Kratzer's (53). A Kratzer oscillator needs harmonic mean $[(1-1 / 2 p q v)(1+1 / 2 p q v)]=$ $\left(1-1 / 4 p^{2} q^{2} v^{2}\right)$, in line with a harmonic oscillator. A normalization is needed for a parabola $(a-x)^{2}=$ $a^{2}(1-x / a)^{2}$, where $x$ can be adapted by factor $1 / a$ to restore (52) or (53). The accuracy of a second-order fit in (53) for $\mathrm{H}_{2}$ is at maximum for $p=0.83795$.

The second-order fits for plots of levels versus $\delta_{\mathrm{Du}}(52)$ and $\delta_{\mathrm{Kr}} / 0.83795$ (53) in Figure 2 are

$$
\begin{aligned}
E_{\delta(D u)}= & -40971.3574 \delta_{\mathrm{Du}}^{2}+78614.1312 \delta_{\mathrm{Du}} \\
& -161.1126 \mathrm{~cm}^{-1}, \\
E_{\delta(K r)}= & -40754.1814 \delta_{\mathrm{Kr}}^{2}+76766.2419 \delta_{\mathrm{Kr}} \\
& -3.56576 \mathrm{~cm}^{-1},
\end{aligned}
$$

with respective goodness of fit of $R^{2}=0.9998627$ for (54) and $R^{2}=0.9999999$ for (55). Although all coefficients are as theoretically expected, they do not

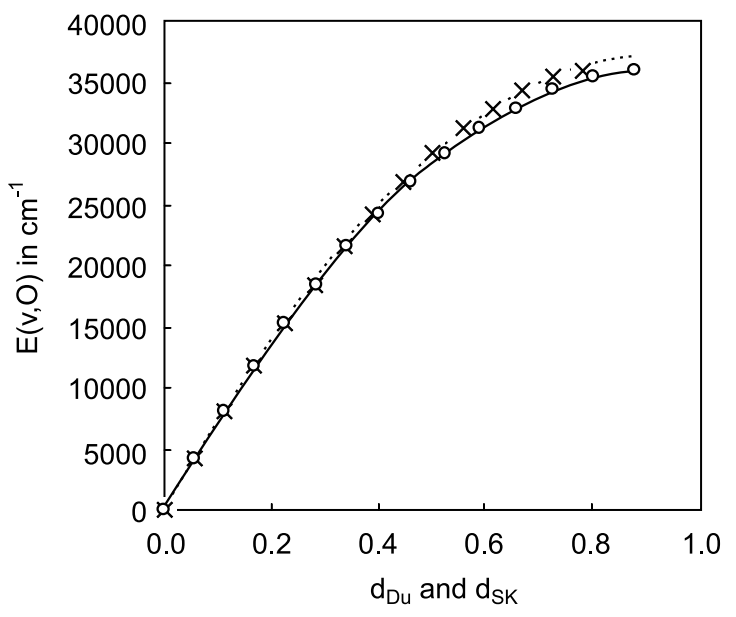

Fig. 2. Plot of $E(v, 0)$ versus Dunham $d_{\text {DU }}$ (curve $\times$ ) and Kratzer $d_{\text {SK }}$ (curve $\circ$ ) variables with second-order fits.

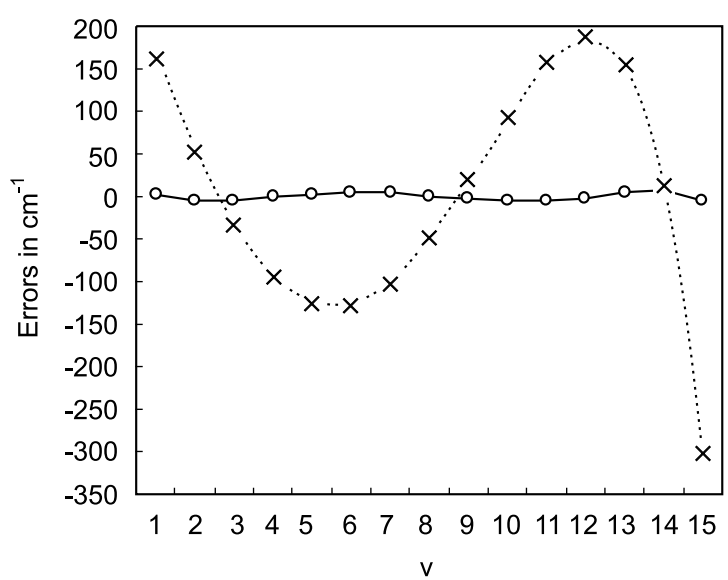

Fig. 3. Errors for $E(v, 0)$ for $\mathrm{H}_{2}$ (in $\mathrm{cm}^{-1}$ ) with second-order fits for Dunham $(\times)$ and Kratzer $(\circ)$ oscillators.

give a normalized parabola (see also Section 6.3). Errors for (54) and (55) show great differences, as shown in Table 2 and Figure 3. Errors of $0.021 \%$ for (55) almost vanish when compared with Dunham's: they are 30 times smaller than $0.54 \%$ for (54) and 530 times smaller for $\mathrm{HO}(1)$, with errors of $1840 \mathrm{~cm}^{-1}$ (see Section 2). Kratzer's second-order fit is even better than Dunham's fourth-order fit, with errors of $7 \mathrm{~cm}^{-1}$ (see Table 2). Errors of $3.5 \mathrm{~cm}^{-1}$ in Kratzer's theory compare with those of $3.2 \mathrm{~cm}^{-1}$ in early QM [10].

\subsection{Parabolic Behaviour: Covalent $\mathrm{H}_{2}$ Bond Energy $D_{\mathrm{e}}$ from Ionic Energy $D_{\text {ion }}$}

Covalent $\mathrm{H}_{2}$ bond energy $D_{\mathrm{e}}$ is obtained directly from ionic bond energy $D_{\text {ion }}$ by making (54) and (55) 


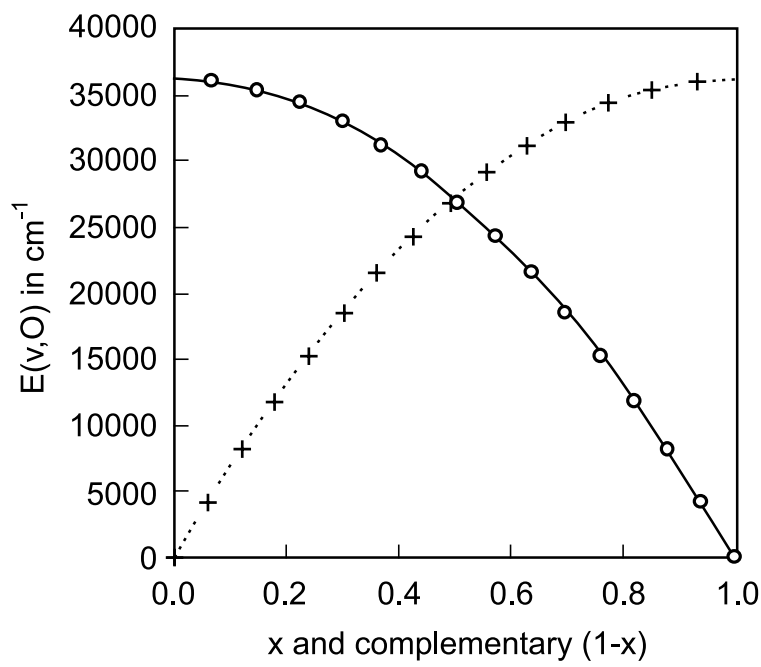

Fig. 4. Energy levels $E(v, 0)$ for $\mathrm{H}_{2}\left(\right.$ in $\left.\mathrm{cm}^{-1}\right)$ with Kratzer parabola (59) versus $x$ (curve + ) and with (57) versus complementary $1-x$ (curve $\operatorname{circ}$ ), giving $D_{\mathrm{e}}$ as intercept (see text).

a perfect parabola $\left(1-\delta^{\prime}\right)^{2}=\left(1-2 \delta^{\prime}+\delta^{\prime 2}\right)$. Coefficient ratio $40754 \cdot 1814 /(1 / 2 \cdot 76766 \cdot 2419)$ in $(55)$ gives $\delta_{\mathrm{Kr}}^{\prime}=1.061774 \delta_{\mathrm{Kr}}$ and $\delta_{\mathrm{Du}}^{\prime}=1.042341 \delta_{\mathrm{Du}}$ for (54). Plotting energies versus variables $x$ and $1-x$ as in Figure 4 gives well depth or covalent bond energy $D_{\mathrm{e}}$ as intercept in

$$
\begin{aligned}
E_{x(\mathrm{Du})}=[ & -37710.3788 x^{2}-0.0000 x \\
& +37549.2663] \mathrm{cm}^{-1}, \\
E_{x(\mathrm{Kr})}=[ & -36150.0077 x^{2}+0.0000 x \\
& +36146.4419] \mathrm{cm}^{-1} .
\end{aligned}
$$

The covalent bond energy $D_{\mathrm{e}}$ of $\mathrm{H}_{2}$, is $37549.27 \mathrm{~cm}^{-1}$ for Dunham's theory (error 3.96\%) but

$$
D_{\mathrm{e}}=36146.442 \mathrm{~cm}^{-1}
$$

for Kratzer's theory (error $0.078 \%$ ). Result (57) is illustrative for the behaviour of a linear Coulomb term, made invisible by complementarity. In fact,

$$
\begin{aligned}
E_{\delta^{\prime}}=[ & -36150.0077 \delta^{\prime 2}+72300.0154 \delta^{\prime} \\
& -3.5658] \mathrm{cm}^{-1}
\end{aligned}
$$

gives $72300.0154 \equiv 2,36150.0077$ as required. This ionic term in (59) gives covalent $D_{\mathrm{e}}$ (58) within $0.078 \%$ of observed $D_{\mathrm{e}}=36118.3 \mathrm{~cm}^{-1}$, better than early $\mathrm{QM}^{7}$ [24].

\footnotetext{
${ }^{7}$ In 1927, Heitler and London obtained $r_{0}=0.80 \AA, \omega_{\mathrm{e}}=$ $4800 \mathrm{~cm}^{-1}$, and $D_{\mathrm{e}}=3.14 \mathrm{eV}$ or $25300 \mathrm{~cm}^{-1}$ [24].
}

\section{Internal $\mathrm{H}_{2}$ Symmetries}

Since bisecting $\mathrm{H}_{2}$, allowed by stoichiometry or mass conservation, gives two hydrogen atoms, we must verify whether one is either an exact copy ( $P$ even) or a mirror image ( $P$ odd) of the other. $\mathrm{CP}$-effects in $\mathrm{H}_{2}$ are available by the differences between them or the ratios of \pm 1 and the form factor $\pm A_{\mathrm{r}}$ (23), related to the errors in Table 2. $C$ is a discrete symmetry \pm 1 , whereas $P$ is a geometry dependent (continuous) symmetry $\pm x$, where $x$ may vary with the $\mathrm{H}_{2}$ structure, on which the form factor $A_{\mathrm{r}}$ gives evidence. Errors for bands $T_{\mathrm{v}}$ are those for levels in Table 2, multiplied by $E_{\mathrm{V}} /\left(T_{0.0}+E_{\mathrm{V}}\right)$, where $T_{0.0}$ is $90203.52 \mathrm{~cm}^{-1}$ or $90206.1 \mathrm{~cm}^{-1}$ [12]. This gives an average error for $T_{\mathrm{V}}$ of only $0.005 \%$. Whatever and however complicated the $3 \mathrm{D} \mathrm{H}_{2}$ structure, its geometry remains constant (within $0.005 \%$ ) according to the Kratzer bond theory. The second-order fit for observed terms,

$$
\begin{aligned}
T_{\mathrm{v}}= & {\left[36150.0077 \delta^{\prime 2}-72300.0154 \delta^{\prime}\right.} \\
& +90207.0858] \mathrm{cm}^{-1}
\end{aligned}
$$

reveals why, after all, a negative ionic attractive Coulomb term $-e^{2} / r_{\mathrm{AB}}$ shows for covalent $\mathrm{H}_{2}$.

The discrete symmetry behind $-A_{\mathrm{r}}$, i.e. attractive $-e^{2} / r$, can only derive from two atom centers in $\mathrm{a}_{2}$ dumbbell, occupied by $+e$ and $-e$. Classically, this is achieved with an ion pair but this requires unlikely ${ }^{8}$ particle transfers at long range. Yet, $-e^{2} / r$ is also obtained with charge inversion $C$ but this transforms an atom in an antiatom, i.e. antihydrogen $\underline{\mathrm{H}}$ [25]. Although $C$-based solutions are the more generic, they are usually not allowed by the prospect of $\mathrm{H} \underline{\mathrm{H}}$ annihilation [25].

Whatever the reason for attractive $-e^{2} / r$ (by virtue of $C$ or by the creation of an ion pair), odd or inversion symmetry $P$ always leads to atom centers with a positive +1 and a negative mark -1 , independent of the sign of charges on these marks. Although $P$ in $\mathrm{H}_{2}$ is reasonably well obeyed (within $0.005 \%$ ) for a fixed $\mathrm{H}_{2}$ geometry (one atom being the mirror image of the other by inversion symmetry), it is still uncertain whether internal symmetry $P$ in $\mathrm{H}_{2}$ is violated or not. To get more certainty about $P$, the origin of the small errors $(<0.005 \%)$ must be retraced. In [26], we show

\footnotetext{
${ }^{8}$ Attractive ionic bonding $-e^{2} / r$ needs a particle transfer between atoms $X$. Large energy gap $I E_{\mathrm{X}}-E A_{\mathrm{X}}\left(I E_{\mathrm{X}}\right.$ and $E A_{\mathrm{X}}$ are ionization energy and electron affinity), makes particle transfers in ionic bonding, improbable at long range $r \gg r_{0}$.
} 
that this origin resides in an extra $\mathrm{H}_{2}$ symmetry, not yet discussed.

\section{Discussion}

(i) A connection between $D_{\text {ion }}$ and $D_{\text {e }}$ affects the theory of the chemical bond: ionic bonding rationalizes covalent bonding in $\mathrm{H}_{2}$. Reduced $\mu_{\text {ion }}=1 / 2 m_{\mathrm{H}}[1-$ $\left.\left(m_{\mathrm{e}} / m_{\mathrm{H}}\right)^{2}\right]$ differs from $\mu_{\text {cov }}=1 / 2 m_{\mathrm{H}}$ by only 3 . $10^{-5} \%$. Resonance between ions $\left[\mathrm{H}^{+} \mathrm{H}^{-} ; \mathrm{H}^{-} \mathrm{H}^{+}\right]$ avoids a permanent dipole moment [25].

(ii) Magnitude and sign of the term in $A_{\mathrm{r}}$ show that only attractive $V=-e^{2} / r_{\mathrm{AB}}$, not mutually exclusive repulsive $+e^{2} / r_{\mathrm{AB}}$ can lead to stable $\mathrm{H}_{2}$. Hence, the $\mathrm{H}_{2}$ dumbbell is anti-symmetrical $\odot \otimes$ or $\otimes \odot$, instead of symmetrical $\odot \odot$ or $\otimes \otimes$. Nevertheless, symmetry based solutions for bonds generate problems with $C$ for neutral $\mathrm{H}$ (formation of antihydrogen $\underline{\mathrm{H}}$ [25]). We discuss some of these issues more in depth in [26].

(iii) Kratzer Coulomb energy $-e^{2} / r_{0}$ is important for universal behaviour and a UF [2,27]. Only scaling by ionic $D_{\text {ion }}[7,27]$ unifies constants of ionic and covalent bonds between all monovalent atoms in the Periodic Table $[2,27-29]$. Why $D_{\text {ion }}$ is a better scaling aid than $D_{\mathrm{e}}[2,27]$ is understood with (60), since $D_{\text {ion }}$, related to $-72300.0154 \delta^{\prime}$, is the first scaling aid $[2,27,28]$, whereas $D_{\mathrm{e}}$ only appears in second instance, see (57) and (59). Difficulties for scaling without $D_{\mathrm{e}}$ [29] are in line with shortcomings of Dunham theory, as reported here.

(iv) Universal behaviour is usually connected with the smooth $G(F)$-plot of Varshni functions $F$ for $\alpha_{\mathrm{e}}$ and $G$ for $\omega_{\mathrm{e}} x_{\mathrm{e}}$, whereby $F$ and $G$ are relate to the Dunham coefficients $a_{1}$ and $a_{2}$ in (3) and $r / r_{0}[2,6,7]$. With Kratzer, higher-order terms in $r$ seem superfluous, whereas higher-order terms in $v$ are generated by the link between $v$ and $r_{0} / r$. The quadratic term $1 / 2\left(e^{2} / r_{0}\right)\left(\omega_{\mathrm{e}} / a_{0}\right)^{2}=\left[0.5 \cdot 4410.17^{2} / 78844.91=\right.$ $123.34 \mathrm{~cm}^{-1}$ agrees with the observed $\mathrm{H}_{2}$ levels. This second-order Kratzer term is close to $\mathrm{H}_{2}$ anharmonicity $\omega_{\mathrm{e}} x_{\mathrm{e}}$ of $123.07 \mathrm{~cm}^{-1}[6,12,22]$. In Dunham's (10) it is related to the fourth-order term and the coefficient $a_{2}$ [2,7].

(v) For chemistry, Morse and Dunham potentials are used more widely than Kratzer's [2] but

[1] J. S. Rigden, Hydrogen, The Essential Element, Harvard University Press, Cambridge 2003.

[2] G. Van Hooydonk, Eur. J. Inorg. Chem., Oct., 1617 (1999). the interest in this function [30] remains justified, as it connects rotations (rolling [31]) with vibrations.

(vi) Recent double photoionization experiments on $\mathrm{H}_{2}$ [32] led to non-Heitler-London ionic states for the $\mathrm{H}_{2}$ ground state, which is in line with the present ionic Kratzer bond theory [33].

(vii) For isotopomers $\mathrm{HD}, D_{2} \ldots$ the results must be as accurate as for $\mathrm{H}_{2}$, since with the approximation $m_{\mathrm{D}}=2 m_{\mathrm{H}}$, similar $r_{0}$ values are obtained for $D_{2}$. This suffices to extend ionic bonding to covalent isotopomers [22, 23], without giving details here.

(viii) Why $\mathrm{H}_{2}$ only shows 14 bands is understood with maximum $v=14.81$ (51), deriving from $m_{\mathrm{H}}$.

(ix) Despite the good performances of an ionic model for covalent $\mathrm{H}_{2}$, it fails, just like early ab initio QM [10], on spectroscopic accuracy. However, errors of $3.5 \mathrm{~cm}^{-1}$ with simple Kratzer theory are almost the same as errors of $3.2 \mathrm{~cm}^{-1}$ with the more complicated and elaborate QM methods [10]. In both cases, errors are larger than with the recent QM [3]. To remove small errors of Kratzer theory for $\mathrm{H}_{2}$, it suffices to adapt symmetries by going over from an achiral to a chiral bond model [26].

\section{Conclusion}

A first-principles Sommerfeld-Kratzer potential accounts quite accurately for $\mathrm{H}_{2}$ vibrations. A simple bond theory exists, which is in line with the existence of a UF. Ionic Kratzer bond theory for covalent $\mathrm{H}_{2}$ differs from the conventional Dunham oscillator and the QM theories. This theory only needs hydrogen mass $m_{\mathrm{H}}$ as input to assess the full $\mathrm{H}_{2}$ vibrational spectrum and links the ionic with the covalent bond energy. A symmetry-adapted Kratzer theory gives spectroscopic accuracy (errors of order $0.00002 \%$ ) for prototypical chiral bond $\mathrm{H}_{2}$, as we will show in [26].

\section{Acknowledgement}

I am in debt to Y.P. Varshni for communicating results prior to publication, to R. J. Le Roy for data and to P. Hajigeorgiou and H. G. M. Edwards for encouraging remarks.

[3] J. Wolniewicz, J. Chem. Phys., 103, 1792 (1995) and references therein.

[4] W. Kolos and L. Wolniewicz, J. Chem. Phys. 49, 404 (1968). 
[5] A. A. Svidzinsky, M. O. Scully, and D. R. Herschbach, PNAS, 102, 11985 (2005); A. A. Svidzinsky, S. A. Chin, and M. O. Scully, Phys. Lett. A 355, 373 (2006); D. Ben-Amotz, J. Phys. Chem. B 110, 19861 (2006).

[6] Y. P. Varshni, Chem. Phys. 342, 297 (2007).

[7] Y. P. Varshni, Rev. Mod. Phys. 29, 664 (1957).

[8] J. L. Dunham, Phys. Rev. 41, 713 (1932).

[9] Y.S. Kim and M.E. Noz, arxiv:quant-ph/0411017.

[10] W. Kolos and L. Wolniewicz, J. Mol. Spect. 54, 303 (1975).

[11] E. Schrödinger, Ann. Phys. 80, 437 (1926).

[12] I. Dabrowski, Can. J. Phys. 62, 1639 (1984).

[13] P. M. Morse, Phys. Rev. 34, 57 (1929).

[14] D. Ter Haar, Phys. Rev. 70, 222 (1946).

[15] G. Simons and J. M. Finlan, Phys. Rev. Lett. 33, 131 (1974).

[16] A. Sommerfeld, Ann. Phys. (Berlin), 50, 1 (1916); La constitution de l'atome et les raies spectrales, $3 \mathrm{~d}$ ed., Blanchard, Paris 1923 ( $1^{\text {st }}$ ed., Atombau und Spektrallinien, Vieweg \& Sohn, Braunschweig 1919).

[17] G. Van Hooydonk, arxiv:physics/012141.

[18] A. Kratzer, Z. Phys. 3, 289 (1920); Ann. Phys. 67, 127 (1922).

[19] W. Kossel, Ann. Phys. 49, 229 (1916); Z. Phys. 23, 403 (1924).

[20] E. Fues, Ann. Phys. 80, 376 (1926).

[21] P. J. Mohr and B. N. Taylor, Rev. Mod. Phys. 77, 1 (2005); http://physics.nist.gov/constants.

[22] K. P. Huber, G. Herzberg, Molecular Spectra, Molecular Structure: Constants of Diatomic Molecules, Vol. IV, Van Nostrand-Reinhold, New York 1979.

[23] R. J. Le Roy and M. G. Barwell, Can. J. Phys. 53, 1983 (1975).

[24] W. Heitler and F. London, Z. Phys. 44, 455 (1927).

[25] G. Van Hooydonk, Eur. Phys. J. D 32, 299 (2005).

[26] G. Van Hooydonk and Y. P. Varshni, arxiv:0906.2905.

[27] G. Van Hooydonk, Phys. Rev. Lett. 100, 159301 (2008).

[28] G. Van Hooydonk, Z. Naturforsch. A 37, 710 (1982); ibidem, A 37, 971 (1982).

[29] Y. P. Varshni, Chem. Phys. 353, 32 (2008).

[30] D. Mikulski, M. Molski, and J. Konarski, Phys. Scripta 80, 025002 (2009); S. m. Ikhdair and R. Sever, J. Math. Chem. 45, 1137 (2009); N. Saad, R.L. Hall, and H. Ciftci, Centr. Eur. J. Phys. 6, 717 (2008); S. M.
Ikhdair and R. Sever, Centr. Eur. J. Phys. 6, 697 (2008); S. H. Patil and K. D. Sen, Phys. Lett. A 362, 109 (2007); S. M. Ikhdair and R. Sever, Int. J. Mod. Phys. A 21, 6699 (2006); P. G. Hajigeorgiou, J. Mol. Spectr. 235, 111 (2006); C. A. Singh and D. B. Devi, Int. J. Quant. Chem. 106, 415 (2006); A. R. Matamala, Int. J. Quant. Chem. 89, 129 (2002); G. Van Hooydonk, Spectrochim. Acta A 56, 2273 (2000); D. R. Herrick and S. O'Connor, J. Chem. Phys. 109, 2071 (1998); R. L. Hall and N. Saad, J. Chem. Phys. 109, 2983 (1998); J. Morales, J. J. Pena, G. Ovando, and V. Gaftoi, J. Math. Chem. 21, 273 (1997); I. L. Cooper, Int. J. Quant. Chem. 49, 2 (1994); A. Requena, M. Alacid, A. Bastida, and J. Zuniga, Int. J. Quant. Chem. 52, 165 (1994); M. Bag, M. M. Panja, R. Dutt, and Y. P. Varshni, J. Chem. Phys. 95, 1139 (1991); D. Secrest, J. Chem. Phys. 89, 1017 (1988); J. Phys. Chem. 95, 1058 (1991); C. Amiot, J. Chem. Phys. 93, 8591 (1990); J. Mol. Spectr. 147, 370 (1991); C. G. Diaz, F. M. Fernandez, and E. A. Castro, Chem. Phys. 157, 31 (1991); A. Alijah and G. Duxbury, Mol. Phys. 70, 605 (1990); S. Brajamani and C. A. Singh, J. Phys. A, Math. Gen. 23, 3421 (1990); A. Bastida, J. Zuniga, M. Alacid, A. Requena, and A. Hidalgo, J. Chem. Phys. 93, 3408 (1990); Y. Ergun, H. O. Pamuk, and E. Yurtsever, Z. Naturforsch. A 45, 889 (1990); J. M. Frances, M. Alacid, and A. Requena, J. Chem. Phys. 90, 5536 (1989); A. Requena, J. Zuniga, L. M. Fuentes, and A. Hidalgo, J. Chem. Phys. 85, 3939 (1986); G. Van Hooydonk, Theochem. J. Mol. Struct. 105, 69 (1983); ibidem, 109, 84 (1984); R. N. Kesarwani and Y. P. Varshni, Chem. Phys. Lett. 93, 545 (1982); R. J. Le Roy and R. B. Bernstein, J. Chem. Phys. 52, 3869 (1970)

[31] L. Grill, J Phys. Cond. Mat. 20, 053001 (2008); W. Ho, J. Chem. Phys. 117, 11033 (2002); D. M. Eigler and E. K. Schweizer, Nature 344, 524 (1990).

[32] K. Kreidi, D. Akoury, T. Jahnke, Th. Weber, A. Staudte, M. Schöffler, N. Neumann, J. Titze, L. Ph. H. Schmidt, A. Czasch, O. Jagutzki, R. A. Costa Fraga, R. E. Grisenti, M. Smolarski, P. Ranitovic, C. L. Cocke, T. Osipov, H. Adaniya, J. C. Thompson, M. H. Prior, A. Belkacem, A. L. Landers, H. Schmidt-Böcking, and R. Dörner, Phys. Rev. Lett. 100, 133005 (2008).

[33] G. Van Hooydonk, arxiv:0805.0947. 